\title{
Entangled quantum heat engines based on two two-spin systems with Dzyaloshinski-Moriya anisotropic antisymmetric interaction
}

\author{
Guo-Feng Zhang*† \\ Department of physics, School of sciences, \\ Beijing University of Aeronautics and Astronautics, \\ Xueyuan Road No. 37, Beijing 100083, People's Republic of China
}

\begin{abstract}
We construct an entangled quantum heat engine (EQHE) based on two two-spin systems with Dzyaloshinski-Moriya (DM) anisotropic antisymmetric interaction. By applying the explanations of heat transferred and work performed at the quantum level in Kieus work [PRL, 93, 140403 (2004)], the basic thermodynamic quantities, i.e., heat transferred, net work done in a cycle and efficiency of EQHE are investigated in terms of DM interaction and concurrence. The validity of the second law of thermodynamics is confirmed in the entangled system. It is found that there is a same efficiency for both antiferromagnetic and ferromagnetic cases, and the efficiency can be controlled in two manners: 1 . only by spin-spin interaction $J$ and DM interaction $D ; 2$. only by the temperature $T$ and concurrence $C$. In order to obtain a positive net work, we need not entangle all qubits in two two-spin systems and we only require the entanglement between qubits in a two-spin system not be zero. As the ratio of entanglement between qubits in two two-spin systems increases, the efficiency will approach infinitely the classical Carnot one. An interesting phenomenon is an abrupt transition of the efficiency when the entanglements between qubits in two two-spin systems are equal.
\end{abstract}

PACS numbers: 03.67.Hk; 03.65.Ud; 75.10.Jm

\footnotetext{
* Corresponding author.

$\dagger$ Email: gf1978zhang@buaa.edu.cn
} 


\section{INTRODUCTION}

The dominating feature of an industrial society is its ability to utilize, whether for wise or unwise ends, sources of energy other than the muscles of men or animals. Except for waterpower, where mechanical energy is directly available, most energy supplies are in the form of potential energy of molecular or nuclear aggregations. In chemical or nuclear reactions, some of this potential energy is released and converted to random molecular kinetic energy. Heat can be withdrawn and utilized for heating buildings, for cooking, or for maintaining a furnace at a high temperature in order to carry out other chemical or physical processes. But to operate a machine, one of the problems of the mechanical engineers is to withdraw heat from a high-temperature source and convert as large as a fraction as possible to mechanical energy. We can solve the problems by using heat engines which can extract energy from its environment in the form of heat and do useful work. We consider for simplicity a heat engine in which the so-called "working substance" is carried through a cycle process, that is, a sequence of processes in which it eventually returns to its original state. All the heat engines absorb heat from a source at a high temperature, perform some mechanical work, and reject heat at a lower temperature. Consider a heat engine operating in a cycle over and over again and let $Q_{h}$ and $Q_{l}$ stand for the heats absorbed and rejected by the working substance per cycle. The net heat absorbed is $Q=Q_{h}+Q_{l}$. The useful output of the engine is the net work $W$ done by the working substance, and from the first law: $W=Q=Q_{h}+Q_{l}$. The heat absorbed is usually obtained from the combustion fuel. The heat rejected ordinarily has no economic value. The thermal efficiency of a cycle is defined as the ratio of the useful work to the heat absorbed: $\eta_{T}=W / Q_{h}=\left(Q_{h}+Q_{l}\right) / Q_{h}$. Because of friction losses, the useful work delivered by an engine is less than the work $W$, and the overall efficiency is less than the thermal efficiency. A Carnot cycle, in which all the heat input is supplied at a single high temperature and all the heat output is rejected at a single lower temperature, has an efficiency less than or equal to the Carnot efficiency $\eta_{c}=\left(Q_{h}+Q_{l}\right) / Q_{h}=1-T_{l} / T_{h}$, where $T_{h}$ and $T_{l}$ are the temperature of the high-temperature energy source and the low-temperature energy sink, respectively. This is supported by the second law of thermodynamics and numerous experimental evidences.

Quantum heat engines (QHEs), in contrast, operated by passing quantum matter through a closed series of quantum adiabatic processes and energy exchanges with heat baths, re- 
spectively, at different stages of a cycle. Kieu[1] constructed a quantum heat engine (QHE) which is a two-level quantum system and undergoes quantum adiabatic process and energy exchanges with heat baths at different stages in a work cycle. Armed with this class of QHE and the interpretations of heat transferred and work performed at the quantum level, he clarified some important aspects of the second law of thermodynamics. Recently, the physics of semiconductors with a spin-orbit interaction has attracted a lot of attention, as it plays an important role for the emerging field of semiconductor spintronics [2]. Sun et al. investigate the equilibrium property of a mesoscopic ring with a spin-orbit interaction, persistent spin current is studied [3].

However, none of the QHEs mentioned above involve the most extraordinary phenomenon in quantum mechanics, i.e., quantum entanglement [4, 5, 6, 7, 8]. To enrich research in QHEs, an entangled QHE and the investigation about the influence of entanglement on its thermodynamic characteristics should be considered. The entanglement of quantum spin systems at finite temperatures has been extensively studied for various interaction styles[9, 10, 11, 12]. Recently, Zhang et al.[13] extended Kieu's work by considering the quantum engine with a two-qubit (isotropic) Heisenberg XXX spin system as the working substance. The spin is subject to a constant external magnetic field. The purpose of their paper is to analyze the effect of quantum entanglement on the efficiency of the quantum engine, but only a constant external magnetic field and entanglement was considered in that study. Also, they only investigated the antiferromagnetic coupling case, that is incomplete. For a ferromagnetic coupling spin chain, whether quantum heat engine is available or not deserve investigation. Moreover in the theoretical analysis we think it is very interesting to investigate the effects of spin-orbit coupling on the basic thermodynamic quantities and should be included. In other words, quantum heat engine is a new physics concept, spin-orbit coupling in spin system plays an important role in the preparation of quantum apparatus. The effect of spin-orbit coupling on quantum heat engine is a very interesting topic and deserves studying. In this paper, We construct an entangled quantum heat engine (EQHE) based on two two-spin systems with a kind of spin-orbit coupling interaction. The positive net work condition in terms of spin-orbit interaction and concurrence will be given. The validity of the second law of thermodynamics at the quantum level is again confirmed. 


\section{ENTANGLED QUANTUM HEAT ENGINE BASED ON TWO TWO-SPIN SYSTEMS WITH DM INTERACTION}

Our EQHE is based on the Heisenberg model with DM interaction, which can be described by

$$
H_{D M}=\frac{J}{2}\left[\left(\sigma_{1 x} \sigma_{2 x}+\sigma_{1 y} \sigma_{2 y}\right)+\vec{D} \cdot\left(\overrightarrow{\sigma_{1}} \times \overrightarrow{\sigma_{2}}\right)\right],
$$

here $J$ is the real coupling coefficient and $\vec{D}$ is the DM vector coupling. The DM anisotropic antisymmetric interaction arises from spin-orbit coupling [14, 15]. The coupling constant $J>0$ corresponds to the antiferromagnetic case and $J<0$ to the ferromagnetic case. For simplicity, we choose $\vec{D}=D \vec{z}$, then the Hamiltonian $H_{D M}$ becomes

$$
\begin{aligned}
H_{D M} & =\frac{J}{2}\left[\sigma_{1 x} \sigma_{2 x}+\sigma_{1 y} \sigma_{2 y}+D\left(\sigma_{1 x} \sigma_{2 y}-\sigma_{1 y} \sigma_{2 x}\right)\right] \\
& =J\left[(1+i D) \sigma_{1+} \sigma_{2-}+(1-i D) \sigma_{1-} \sigma_{2+}\right] .
\end{aligned}
$$

Without loose of generality, we define $|0\rangle(|1\rangle)$ as the ground (excited) state of a two-level particle. The eigenvalues and eigenvectors of $H_{D M}$ are given by

$$
\left|\Psi^{1}\right\rangle=|00\rangle,\left|\Psi^{2}\right\rangle=|11\rangle,\left|\Psi^{3}\right\rangle=\frac{1}{\sqrt{2}}\left\{|01\rangle+e^{i \theta}|10\rangle\right\},\left|\Psi^{4}\right\rangle=\frac{1}{\sqrt{2}}\left\{|01\rangle-e^{i \theta}|10\rangle\right\},
$$

with $E_{1}=E_{2}=0, E_{3}=J \sqrt{1+D^{2}}=-E_{4}$ and $\theta=\arctan D$. As the thermal fluctuation (temperature $T$ ) is introducing into the system, the state of a typical solid-state system at thermal equilibrium (temperature $T$ ) is $\rho(T)=\frac{1}{Z} e^{-\beta H_{D M}}$, where $H_{D M}$ is the Hamiltonian, $Z=\operatorname{tre}^{-\beta H_{D M}}$ is the partition function, $\beta=1 /(k T)$ and $k$ is Boltzmann's constant, for simplicity, we write $k=1$. The entanglement between two qubits in this model is known as [16]:

$$
C= \begin{cases}\frac{\sinh \left(|J| \sqrt{1+D^{2}} / T\right)-1}{\cosh \left(|J| \sqrt{1+D^{2}} / T\right)+1}, & \text { if }|J| \sqrt{1+D^{2}}>T \operatorname{arcsinh}(1) \\ 0, & \text { if }|J| \sqrt{1+D^{2}} \leq T \operatorname{arcsinh}(1) .\end{cases}
$$

Here, we use concurrence directly as the measurement of entanglement, since there is a one-to-one correspondence between the entanglement of formation and concurrence [17, 18]. The concurrence $C=0$ indicates the vanishing entanglement. The critical temperature $T_{c}=\frac{|J| \sqrt{1+D^{2} / T}}{\operatorname{arcsinh}(1)} \approx 1.1346|J| \sqrt{1+D^{2}}$ above which the concurrence is zero.

Based on the Kieus explanations of heat transferred and work performed at the quantum level [1]: The expectation value of the measured energy of a quantum system with discrete 
energy levels is: $U=<E>=\sum_{i} p_{i} E_{i}$, where $E_{i}$ are the energy levels and $p_{i}$ are the corresponding occupation probabilities. Infinitesimally, $d U=\sum_{i}\left\{p_{i} d E_{i}+E_{i} d p_{i}\right\}$, from which we can obtain the following identifications for infinitesimal heat transferred $\bar{d} Q$ and work done $\bar{d} W$

$$
\bar{d} Q:=\sum_{i} E_{i} d p_{i}, \bar{d} W:=\sum_{i} p_{i} d E_{i}
$$

Mathematically speaking, these are not total differentials but are path dependent. These expressions interpret heat as a change in the occupation probabilities but not in the distribution of the energy eigenvalues themselves; and work done as the redistribution of the energy eigenvalues but not of the occupation probabilities of each energy level [19]. Thus, $d U=\bar{d} Q+\bar{d} W$, which is an expression of the first law of thermodynamics.

To set the stage, we describe the four quantum thermodynamic processes of the quantum cycle. In the following, we consider as working substance a bipartite quantum system consisting of two subsystems, $A$ and $B$, with the above Hamiltonian (2). A cycle of the quantum heat engine consists of four stages:

$\langle 1\rangle$ The system has the probability $p_{i 0}(\mathrm{i}=1,2,3,4)$ to be in each of its four eigenstates, respectively. By contacting with a heat bath at temperature $T_{h}$ for some time, the occupation probability of each eigenstate becomes $p_{i 1}$. The quantum state of the system is given by the density operator $\rho_{A B}^{1}=\sum_{i} p_{i 1}\left|\Psi^{i 1}\right\rangle\left\langle\Psi^{i 1}\right|,(i=1,2,3,4)$ with $p_{i 1}=\exp \left(-E_{i 1} / k T_{h}\right) / Z_{1}$ and $Z_{1}=\sum_{i} \exp \left(-E_{i 1} / k T_{h}\right)$. In other words, we assume the system is initially in thermal equilibrium with a heat bath at temperature $T_{h}$. In our model, we have $E_{i 1}=E_{i},\left|\Psi^{i 1}\right\rangle=$ $\left|\Psi^{i}\right\rangle$ with $J=J_{1}$ and $D=D_{1}$. Only heat is transferred in this process due to the change in the occupation probability. $\langle 2\rangle$ The system is then isolated from the heat bath and undergoes a quantum adiabatic process, with $J$ changing from $J_{1}$ to $J_{2}$ and $D$ changing from $D_{1}$ to $D_{2}$. Provided the rate of change is sufficiently slow, $p_{i 1}$ 's are maintained throughout according to the quantum adiabatic theorem. At the end of process $\langle 2\rangle$, the system has the probability $p_{i 1}$ in the eigenstate $\left|\Psi^{i 1}\right\rangle$. An amount of work is performed by the system, but no heat is transferred. $\langle 3\rangle$ The system is next brought into some kind of contact with another heat bath at temperature $T_{l}\left(T_{l}<T_{h}\right)$ for some time. After the irreversible thermalization process, the quantum state of the system is given by the density operator $\rho_{A B}^{2}=\sum_{i} p_{i 2}\left|\Psi^{i 2}\right\rangle\left\langle\Psi^{i 2}\right|,(i=$ $1,2,3,4)$ with $p_{i 2}=\exp \left(-E_{i 2} / k T_{l}\right) / Z_{2}$ and $Z_{2}=\sum_{i} \exp \left(-E_{i 2} / k T_{l}\right)$. Here, we have $E_{i 2}=E_{i}$, $\left|\Psi^{i 2}\right\rangle=\left|\Psi^{i}\right\rangle$ with $J=J_{2}$ and $D=D_{2}$. Only heat is transferred in this process to yield a 
change in the occupation probabilities, and the heat transferred is given by $Q_{l}=\sum_{i} E_{i 2}\left(p_{i 2}-\right.$ $\left.p_{i 1}\right)$. $\langle 4\rangle$ The system is removed from the heat bath and undergoes a quantum adiabatic process, with for instance $J$ changing from $J_{2}$ to $J_{1}$ and $D$ changing from $D_{2}$ to $D_{1}$. And the occupation probability of each eigenstate is maintained, that is, $p_{i 2}=p_{i 0}$. An amount of work is performed on the system, but no heat is transferred during the process. So we can know the heat transferred in the first process is $Q_{h}=\sum_{i} E_{i 1}\left(p_{i 1}-p_{i 0}\right)=\sum_{i} E_{i 1}\left(p_{i 1}-p_{i 2}\right)$. From the law of conservation of energy, the net work done by the EQHE in two quantum adiabatic processes is given by $W=Q_{h}+Q_{l}=\sum_{i}\left(E_{i 1}-E_{i 1}\right)\left(p_{i 1}-p_{i 2}\right),(i=1,2,3,4)$. For a actual engine, $W>0$ is required, it means that three possible cases, i.e., $Q_{h}>-Q_{l}>0$, or $Q_{l}>-Q_{h}>0$, or $Q_{h}>0$ and $Q_{l}>0$.

\section{BASIC THERMODYNAMIC QUANTITIES IN TERMS OF DM INTERAC- TION}

After a simple calculation, we can get the heat absorbed $Q_{h}$, the heat refused $Q_{l}$ and the net work

$$
\begin{aligned}
Q_{h} & =\sqrt{1+D_{1}^{2}} J_{1}\left(\tanh \left[\frac{J_{2} \sqrt{1+D_{2}^{2}}}{2 T_{l}}\right]-\tanh \left[\frac{J_{1} \sqrt{1+D_{1}^{2}}}{2 T_{h}}\right]\right), \\
Q_{l} & =\sqrt{1+D_{2}^{2}} J_{2}\left(\tanh \left[\frac{J_{1} \sqrt{1+D_{1}^{2}}}{2 T_{h}}\right]-\tanh \left[\frac{J_{2} \sqrt{1+D_{2}^{2}}}{2 T_{l}}\right]\right), \\
W & =\left(J_{2} \sqrt{1+D_{2}^{2}}-J_{1} \sqrt{1+D_{1}^{2}}\right)\left(\tanh \left[\frac{J_{1} \sqrt{1+D_{1}^{2}}}{2 T_{h}}\right]-\tanh \left[\frac{J_{2} \sqrt{1+D_{2}^{2}}}{2 T_{l}}\right]\right) .
\end{aligned}
$$

Thus, under the postulate $T_{l}<T_{h}$, in order to make $W>0$, we must have

$$
\frac{J_{1} \sqrt{1+D_{1}^{2}}}{T_{h}}<\frac{J_{2} \sqrt{1+D_{2}^{2}}}{T_{l}} \Leftrightarrow T_{h}>T_{l}\left(\frac{J_{1} \sqrt{1+D_{1}^{2}}}{J_{2} \sqrt{1+D_{2}^{2}}}\right) .
$$

for $J_{2} \sqrt{1+D_{2}^{2}}<J_{1} \sqrt{1+D_{1}^{2}}$. If the DM interaction is turned off, Eq.(7) will be replaced by

$T_{h}>T_{l}\left(\frac{J_{1}}{J_{2}}\right)$. These conditions are rigorous in contradistinction to the classical requirement that $T_{h}$ only needs to be larger than $T_{l}$.

The thermal efficiency of a cycle is defined as the ratio of the useful work to the heat absorbed: $\eta_{T}=W / Q_{h}=\left(Q_{h}+Q_{l}\right) / Q_{h}$. After a straight calculation, the efficiency can be given by

$$
\eta=1-\frac{J_{2} \sqrt{1+D_{2}^{2}}}{J_{1} \sqrt{1+D_{1}^{2}}}
$$


We can find that the efficiency is independent of temperature and can be controlled only by spin-spin interaction $J$ and DM interaction $D$. Although this is true, in order to obtain a positive net work, the requirement about temperature Eq.(7) must be satisfied.

\section{THE EFFECT OF CONCURRENCE ON BASIC THERMODYNAMIC QUANTITIES}

The entanglement under our consideration is that of two thermal equilibrium states at the end of stage $\langle 1\rangle$ and stage $\langle 3\rangle$, denoted by $C_{1}$ and $C_{2}$, respectively. They are

$$
\begin{gathered}
C_{1}= \begin{cases}\frac{\sinh \left(\left|J_{1}\right| \sqrt{1+D_{1}^{2}} / T_{h}\right)-1}{\cosh \left(\left|J_{1}\right| \sqrt{1+D_{1}^{2}} / T_{h}\right)+1} & \text { if }\left|J_{1}\right| \sqrt{1+D_{1}^{2}}>T_{h} \operatorname{arcsinh}(1) ; \\
0 & \text { if }\left|J_{1}\right| \sqrt{1+D_{1}^{2}} \leq T_{h} \operatorname{arcsinh}(1)\end{cases} \\
C_{2}= \begin{cases}\frac{\sinh \left(\left|J_{2}\right| \sqrt{1+D_{2}^{2}} / T_{l}\right)-1}{\cosh \left(\left|J_{2}\right| \sqrt{1+D_{2}^{2}} / T_{l}\right)+1} & \text { if }\left|J_{2}\right| \sqrt{1+D_{2}^{2}}>T_{l} \operatorname{arcsinh}(1) ; \\
0 & \text { if }\left|J_{2}\right| \sqrt{1+D_{2}^{2}} \leq T_{l} \operatorname{arcsinh}(1) .\end{cases}
\end{gathered}
$$

Here we will explore the relation between entanglement and basic thermodynamics quantities.

Case1: The two-spin system is antiferromagnetic coupling, i.e., $J>0$. From Eq.(9), we find

$$
\begin{aligned}
& J_{1}=\frac{T_{l}}{\sqrt{1+D_{1}^{2}}} \ln \left(\frac{-1-C_{1}-\sqrt{2\left(1+C_{1}\right)}}{C_{1}-1}\right), \\
& J_{2}=\frac{T_{h}}{\sqrt{1+D_{2}^{2}}} \ln \left(\frac{-1-C_{2}-\sqrt{2\left(1+C_{2}\right)}}{C_{2}-1}\right) .
\end{aligned}
$$

By simple deduction, we can obtain

$$
\begin{aligned}
Q_{h} & =\sqrt{2} \ln \left[\frac{1}{-1+\sqrt{\frac{2}{1+C_{1}}}}\right]\left(\sqrt{1+C_{2}}-\sqrt{1+C_{1}}\right) T_{h}, \\
Q_{l}= & \sqrt{2} \ln \left[\frac{1}{-1+\sqrt{\frac{2}{1+C_{2}}}}\right]\left(\sqrt{1+C_{1}}-\sqrt{1+C_{2}}\right) T_{l}, \\
W= & -\sqrt{2}\left(\sqrt{1+C_{1}}-\sqrt{1+C_{2}}\right)\left(\ln \left[\frac{1}{-1+\sqrt{\frac{2}{1+C_{1}}}}\right] T_{h}-\ln \left[\frac{1}{-1+\sqrt{\frac{2}{1+C_{2}}}}\right] T_{l}\right) .
\end{aligned}
$$

Therefore the positive net work condition indicates two possible situations, i.e.,

$$
\sqrt{1+C_{1}}<\sqrt{1+C_{2}} \quad \text { and } \quad \ln \left[\frac{1}{-1+\sqrt{\frac{2}{1+C_{1}}}}\right] T_{h}>\ln \left[\frac{1}{-1+\sqrt{\frac{2}{1+C_{2}}}}\right] T_{l}
$$



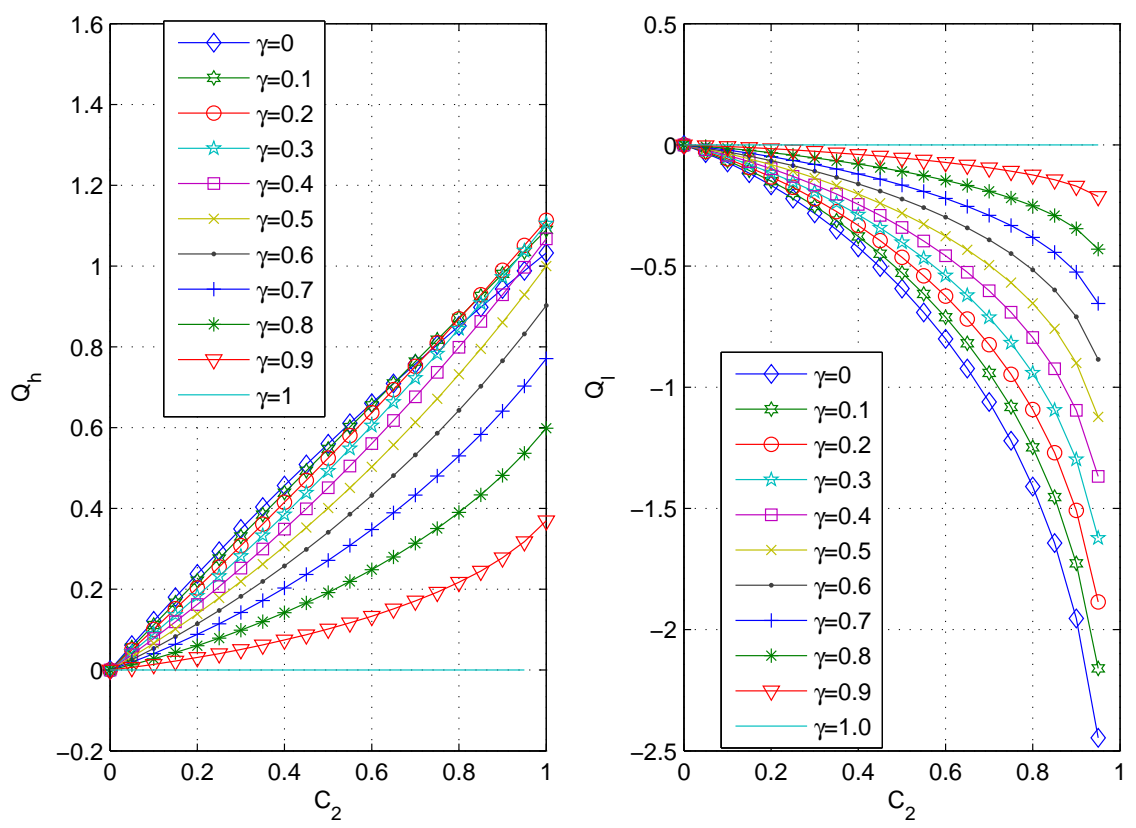

FIG. 1: (Color online) The heat absorbed $Q_{h}$ and the one rejected $Q_{l}$ are plotted vs the concurrence $C_{2}$ for different $\gamma=C_{1} / C_{2}$ and $k T_{h}=2, k T_{l}=1$.

or

$$
\sqrt{1+C_{1}}>\sqrt{1+C_{2}} \quad \text { and } \quad \ln \left[\frac{1}{-1+\sqrt{\frac{2}{1+C_{1}}}}\right] T_{h}<\ln \left[\frac{1}{-1+\sqrt{\frac{2}{1+C_{2}}}}\right] T_{l} \text {. }
$$

Equation (12) can be easily proved incompatible with $T_{h}>T_{l}$, that is, case $Q_{h}<0$ and $Q_{l}>0$ is impossible. So far we have clarified case $Q_{h}>-Q_{l}>0$ is the only possible case. It is shown that the second law of thermodynamics is valid in this case.

Case2: The two-spin system is ferromagnetic coupling, i.e., $J<0$. From Eq.(9), we find

$$
\begin{aligned}
& J_{1}=\frac{T_{l}}{\sqrt{1+D_{1}^{2}}} \ln \left(\frac{-1-C_{1}+\sqrt{2\left(1+C_{1}\right)}}{C_{1}+1}\right), \\
& J_{2}=\frac{T_{h}}{\sqrt{1+D_{2}^{2}}} \ln \left(\frac{-1-C_{2}+\sqrt{2\left(1+C_{2}\right)}}{C_{2}+1}\right) .
\end{aligned}
$$

Similarly, we can obtain

$$
\begin{aligned}
Q_{h} & =\sqrt{2} \ln \left[-1+\sqrt{\frac{2}{1+C_{1}}}\right]\left(\sqrt{1+C_{1}}-\sqrt{1+C_{2}}\right) T_{h}, \\
Q_{l} & =\sqrt{2} \ln \left[-1+\sqrt{\frac{2}{1+C_{2}}}\right]\left(\sqrt{1+C_{2}}-\sqrt{1+C_{1}}\right) T_{l}, \\
W & =\sqrt{2}\left(\sqrt{1+C_{1}}-\sqrt{1+C_{2}}\right)\left(\ln \left[-1+\sqrt{\frac{2}{1+C_{1}}}\right] T_{h}-\ln \left[-1+\sqrt{\frac{2}{1+C_{2}}}\right] T_{l}\right) .
\end{aligned}
$$




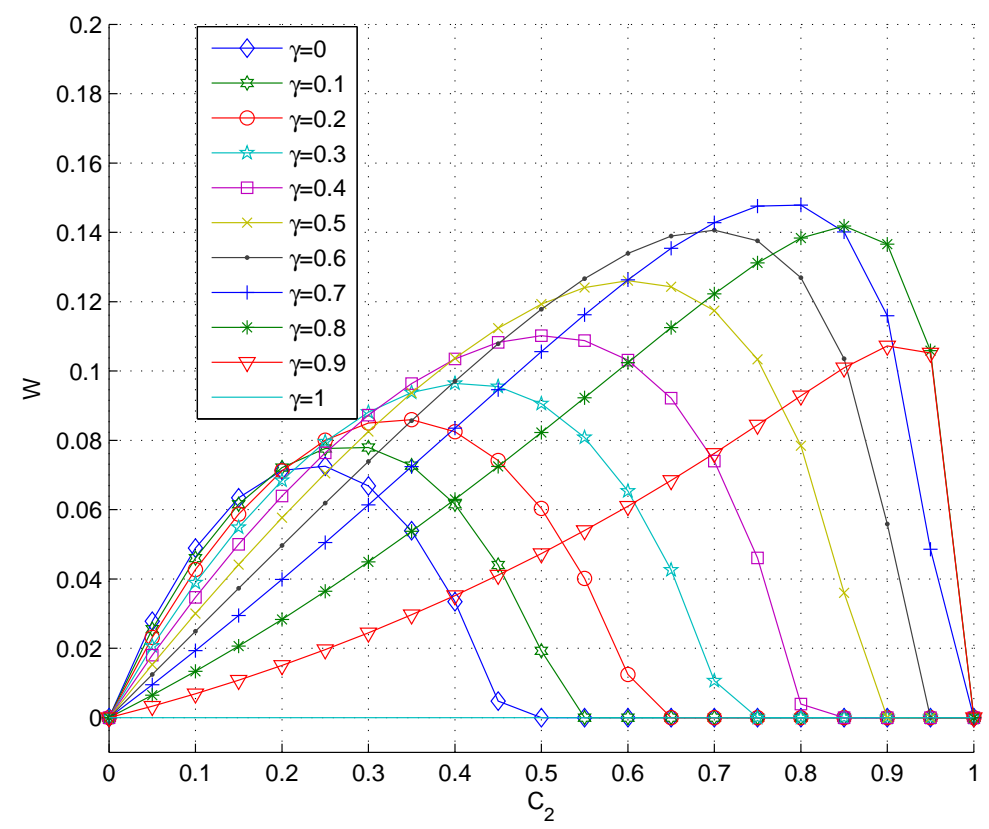

FIG. 2: (Color online) The net work done by the EQHE is plotted vs the concurrence $C_{2}$ for different $\gamma=C_{1} / C_{2}$ and $k T_{h}=2, k T_{l}=1$.

In order to make $W>0$, we must have

$$
\sqrt{1+C_{1}}<\sqrt{1+C_{2}} \quad \text { and } \quad \ln \left[-1+\sqrt{\frac{2}{1+C_{1}}}\right] T_{h}<\ln \left[-1+\sqrt{\frac{2}{1+C_{2}}}\right] T_{l},
$$

or

$$
\sqrt{1+C_{1}}>\sqrt{1+C_{2}} \quad \text { and } \quad \ln \left[-1+\sqrt{\frac{2}{1+C_{1}}}\right] T_{h}>\ln \left[-1+\sqrt{\frac{2}{1+C_{2}}}\right] T_{l} .
$$

It is obvious that Eq.(16) is impossible for $T_{h}>T_{l}$. In fact, we can see that Eq.(11)(Eq.(12)) is the same with Eq.(15)(Eq.(16)). Thus, we can conclude that the second law of thermodynamics is also valid in this case.

Variations of $Q_{h}, Q_{l}$ and the net work $W$ with $C_{2}$ are given in fig.1 and fig.2 for antiferromagnetic coupling. Where we define $\gamma=C_{1} / C_{2}$ as a measurement of the difference of $C_{1}$ and $C_{2}$. From fig.1 and fig.2, We can see the engine undergoes a trivial cycle since $Q_{h}=Q_{l}=W=0$ for $\gamma=1$. That means, in order to make the engine be useful, the entanglement between qubits for the two two-spin systems can not be equal. This result accords with Eq.(11) and Eq.(15). For $\gamma=0$, i.e., $C_{1}=0$, the engine is available since $W>0$ for a range of $C_{2}$. So long as $\gamma \neq 1$, the range of $C_{2}$ in which $W>0$ will be broaden as $\gamma$ increases. The results show that we need not entangle qubits in two-spin system 1 and 

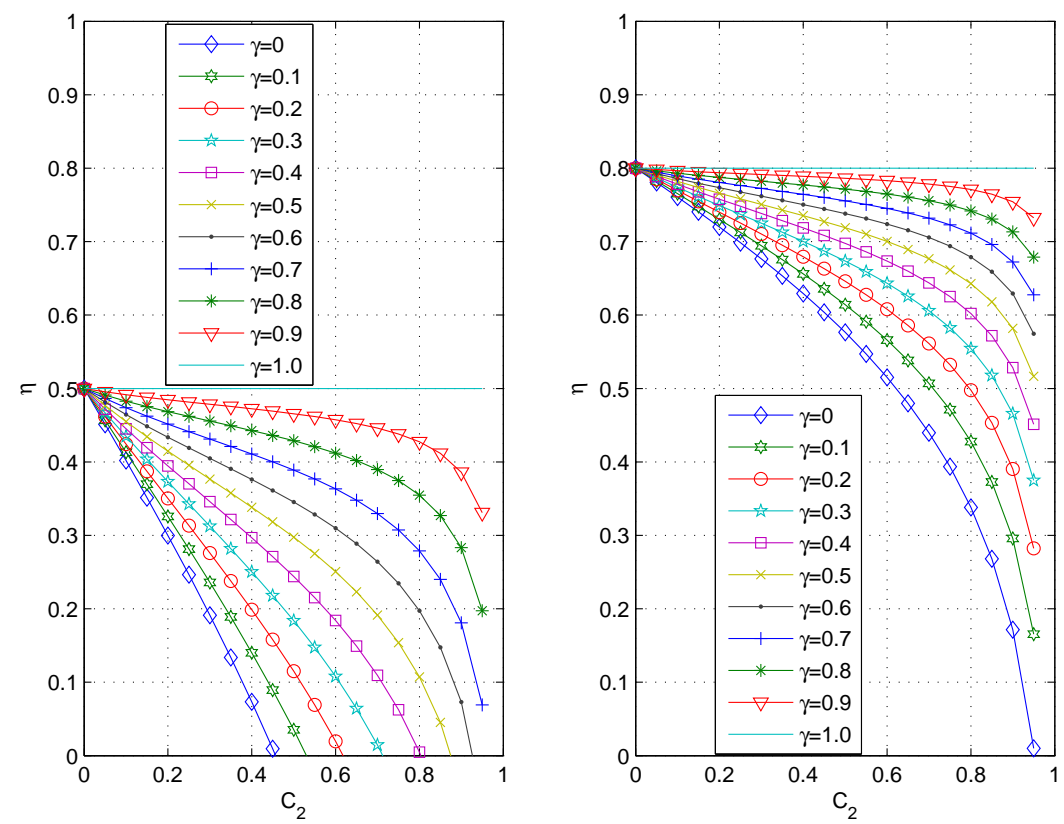

FIG. 3: (Color online) The efficiency is plotted vs the concurrence $C_{2}$ for different $\gamma=C_{1} / C_{2}$. And the left panel for $k T_{h} / k T_{l}=2$, the right panel for $k T_{h} / k T_{l}=5$.

we only require the entanglement in two-spin system 2 not be zero. The entanglement in two-spin system 1 will enhance the validity of the engine.

The efficiency in terms of concurrence can be given by

$$
\eta(J>0)=\eta(J<0)=1-\frac{\ln \left[\frac{1}{-1+\sqrt{\frac{2}{1+C_{2}}}}\right] T_{l}}{\ln \left[\frac{1}{-1+\sqrt{\frac{2}{1+C_{1}}}}\right] T_{h}}, \quad \text { for } \sqrt{1+C_{1}}<\sqrt{1+C_{2}} .
$$

When $C_{2}=C_{1}, \eta(J>0)=\eta(J<0)=0$ since $Q_{h}=Q_{l}=W=0$ from Eqs.(11) and (15). In order to intuitively investigate how entanglement affects the efficiency, we sketch the variation of $\eta$ with $C_{2}$ for different ratio of $C_{1}$ to $C_{2}$ in Fig.3. It is known that for a Carnot cycle, in which all the heat input is supplied at a single high temperature and all the heat output is rejected at a single lower temperature, has an efficiency less than or equal to the Carnot efficiency $\eta_{c}=\left(Q_{h}+Q_{l}\right) / Q_{h}=1-T_{l} / T_{h}$. Here $\eta_{c}$ is never achievable since $C_{1}$ can not be equal to $C_{2}$. From fig. $3, \eta$ increases with $\gamma$ when $C_{2}$ is fixed and it will approach $\eta_{c}$ infinitely. We can also see that $\eta$ decreases with $C_{2}$ and whether it will arrive at zero depends on $\gamma$. The smaller $\gamma$ is, the bigger zero probability $\eta$ has. When $C_{2}=C_{1}$, i.e., $\gamma=1$, we can find $\eta=\eta_{c}$ from fig. 3 , but $Q_{h}=Q_{l}=W=0$ and $\eta$ should be zero. So we say an abrupt transition of the efficiency occurs at $\gamma=1$. Compared the left panel and the 
right one, $\gamma=0$, i.e., $C_{1}=0, \eta$ can not be zero for a range of $C_{2}$ and the range will be enlarged as the ratio $k T_{h} / k T_{l}$ increases. For a same $\gamma, k T_{h} / k T_{l}$ must be greater in order to get a higher efficiency. This result is consistent with the second law of thermodynamics. In other words, the second law of thermodynamics is proved to hold all the time even when entanglement is indeed involved.

\section{CONCLUSIONS}

In conclusion, by quoting the quantum interpretations of heat and work from Ref.[1], we construct an entangled quantum heat engine (EQHE) based on two two-spin systems with Dzyaloshinski-Moriya (DM) anisotropic antisymmetric interaction. The basic thermodynamic quantities, i.e., the heat transferred and the work done in a cycle, and the efficiency of EQHE are investigated in terms of DM interaction and concurrence. The condition for a positive work is given. Four features of main results can be found in this paper. First, the efficiency can be controlled only by spin-spin interaction and DM interaction, it is independent of the temperature. Second, we need not entangle qubits in two-spin system 1 and we only require the entanglement in two-spin system 2 not be zero in order to obtain a positive net work. The entanglement in two-spin system 1 will enhance the validity of the engine. Third, for the same $\gamma$, the ratio $k T_{h} / k T_{l}$ must be greater in order to get a higher efficiency. Fourth, an interesting phenomenon is an abrupt transition of the efficiency when $C_{1}=C_{2}$. These results in the paper are consistent with the second law of thermodynamics. In other words, the second law of thermodynamics is shown to be valid in this entangled system.

\section{ACKNOWLEDGEMENTS}

It is a pleasure to thank the reviewer and editor for their many fruitful discussions about the topic. This work was supported by the National Natural Science Foundation of China (Grant No. 10604053) and Beihang Lantian Project.

[1] T. D. Kieu, Phys. Rev. Lett. 93, 140403 (2004); T. D. Kieu, Eur. Phys. J. D 39, 115 (2006).

[2] I. Zutic, J. Fabian, and S. Das Sarma, Rev. Mod. Phys. 76, 323 (2004). 
[3] Q. F. Sun, X. C. Xie and J. Wang, Phys. Rev. Lett. 98, 196801 (2007).

[4] A. Einstein, B. Podolsky and N. Rozen, Phys. Rev., 47, 777 (1935).

[5] E. Schrdinger, Naturwiss. 23, 807 (1935).

[6] Lecture by Preskill, Quantum Information and Quantum Computation (Proc. Cambridge Philos. Soc. 32:446-452, 1936).

[7] R. Horodecki, P. Horodecki, M. Horodecki and K. Horodecki, arXiv:quant-ph/0702225, (2007).

[8] C. H. Bennett and D. P. DiVincenzo, Nature (London) 404, 247 (2000).

[9] M. C. Arnesen, S. Bose, and V. Vedral, Phys. Rev. Lett. 87, 277901 (2001).

[10] D. Gunlycke et al, Phys. Rev. A 64, 042302 (2001).

[11] X. Wang, and P. Zanardi, Phys. Lett. A 301 (1-2), 1 (2002).

[12] M. Asoudeh, and V. Karimipour, Phys. Rev. A 70, 052307 (2004); Phys. Rev. A,71, 022308 (2005).

[13] T. Zhang, W.-T. Liu, P.-X. Chen, and C.-Z. Li, Phys. Rev. A 75, 062102 (2007).

[14] I. Dzyaloshinskii, J. Phys. Chem. Solids 4, 241 (1958).

[15] T. Moriya, Phys. Rev. Lett. 4, 228 (1960); T. Moriya, Phys. Rev. 117, 635 (1960); T. Moriya, Phys. Rev. 120, 91 (1960).

[16] X. G. Wang, Phys. Lett. A 281, 101 (2001).

[17] S. Hill and W. K. Wootters, Phys. Rev. Lett. 78, 5022 (1997).

[18] W. K. Wootters, Phys. Rev. Letts. 80, 2245 (1998).

[19] E. Schrodinger, Statistical Thermodynamics (Dover, New York, 1998). 